\title{
THE APPLiCATION OF ARTIFICIAL VECTOR FiELDS FOR MOTION CONTROL OF A HETEROGENEOUS MODULAR MOBILE ROBOT
}

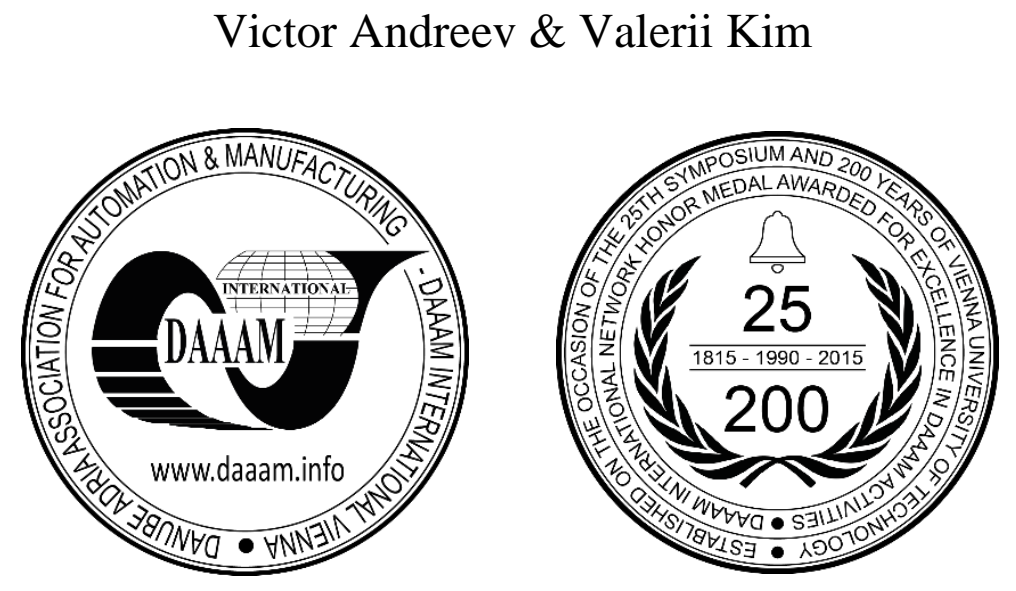

This Publication has to be referred as: Andreev, V[ictor] \& Kim, V[alerii] (2017). The Application of Artificial Vector Fields for Motion Control of a Heterogeneous Modular Mobile Robot, Proceedings of the 28th DAAAM International Symposium, pp.0635-0644, B. Katalinic (Ed.), Published by DAAAM International, ISBN 978-3-902734-11-2, ISSN 1726-9679, Vienna, Austria

DOI: $10.2507 / 28$ th.daaam.proceedings.090

\begin{abstract}
This paper presents a novel method for motion realization of differential drive mobile robot along a complex trajectory using two-dimensional vector fields (artificial forces). The method allows robot to move along trajectories that similar to B-splines without their preparatory computation. This is performed by using the given sequence of points (intermediate goal points) and manipulation of vector fields that defined by Gaussian functions. The feature of the method realization is as follows in its application in the transport module of a heterogeneous modular mobile robot which modules are functionally complete electronic or mechatronic devices. A functional completeness in this case means that every module must perform the function that built in its engineering implementation and control system by itself. The transport module realizes its functions upon receipt control goals (parameters of position vector) from module-supervisor that is part of modular mobile robot. In this case the transport module computes motion trajectory independently. Therefore a method of information interaction between the transport module and a module-supervisor is considered in this paper. The transport module performance under control of a module-supervisor is viewed by the example of mobile robotics typical tasks: motion in a known and unknown environment.
\end{abstract}

Keywords: mobile robot; heterogeneous modular robot; differential drive; motion planning; trajectory following

\section{Introduction}

At the present time mobile robots are used for different tasks and involved in various areas of activities [1-2]. Many researches consider the wheeled mobile robot control problems such as motion and path planning [3-6]. There are many fruitful results in these topics that are applied to different kinds of robots. One of the most challenging problems is a trajectory planning of mobile robot and path following [5-6]. In the most cases robot controllers for trajectory or path following guarantee the minimal tracking errors. But these controllers involve complex and sophisticated control laws that require more powerful on-board computers of mobile robots. Furthermore tracking controllers are even more complicated for nonholonomic wheeled mobile robots [7]. 
In this paper we present a novel motion planning method for a differential drive mobile robot using two-dimensional vector fields. This method requires that the given path for a mobile robot control system must be specified as a set of the reference points that we will call here the "nodal" points. Using the artificial vector fields mobile robot can move along trajectories like splines in proximity to nodal points. The robot motion in this case is smooth and continuous. Note that no trajectory planning is needed for this method. Our approach lies in a design of the such motion planning controller that allows a differential drive mobile robot to move along complex trajectories without classic tracking controllers.

The second objective of our research is the application of the proposed method to a heterogeneous modular mobile robot. This can be done efficiently because concerned modular robot control system can divide general task to several control goals for each module of the robot. This key feature considered in the fourth section.

\section{Motion planning using vector fields}

The essence of the method. Consider a given path as a set of the nodal points with respect to world coordinate system $O X Y:\left(X_{1}, Y_{1}\right), \ldots,\left(X_{i}, Y_{i}\right), \ldots,\left(X_{n}, Y_{n}\right)=\left(X_{G}, Y_{G}\right)$, where $\left(X_{G}, Y_{G}\right)$ - the goal position coordinates at the end of a path, $n$ - number of points. The robot start position is in the point $\left(X_{0}, Y_{0}\right)$. The robot control system takes this set of the nodal points from some robot path planner at the higher control level. Let us assign the vector field that defines the attractive force $F_{i}$ to each nodal points of the given path (fig. 1).

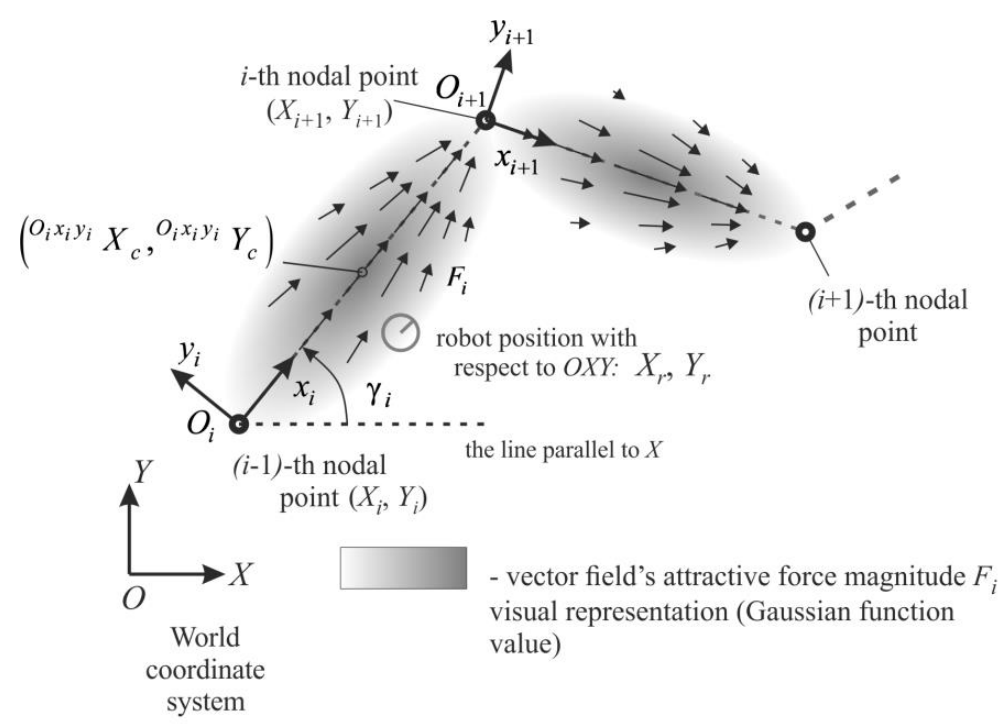

Fig. 1. Vector fields of attractive forces

The $i$-th $(i=\overline{1, n})$ attractive force magnitude $F_{i}$ in each point of the vector field is a continuous function of distance between the robot and the centre of the line segment connecting previous $(i-1)$ and current $i$ nodal points. For this function, we take two-dimensional Gaussian function. Each Gaussian function for the attractive force is rotated along line segment connecting previous $(i-1)$ and current $i$ nodal points. Also a width of Gaussian function along this line segment is larger in comparison to transverse direction. It is needed because closely spaced Gaussian function can influence over robot motion. Considering all these properties we define the next Gaussian function:

$$
F_{i}=F_{0} e^{-\left[\left(w_{x, i}\left({ }^{O_{i} x_{i} y_{i}} X_{r}-{ }^{o_{i} x_{i} y_{i}} X_{c}\right)\right)^{2}+\left(w_{y, i}\left({ }^{O_{i} x_{i} y_{i}} Y_{r}-{ }^{O_{i} x_{i} y_{i}} Y_{c}\right)\right)^{2}\right]},
$$

where $F_{0}$ - the attractive force basis magnitude, ${ }^{O_{i} x_{i} y_{i}} X_{r},{ }^{O_{i} x_{i} y_{i}} Y_{r}$ - the robot coordinates with respect to the turned Gaussian function coordinate system - $O_{i} x_{i} y_{i}$. Here we must mention that robot coordinates is the coordinates of its coordinate system $O^{\prime} x y$ datum $O^{\prime}$. Consider next parameters: ${ }^{O_{i} x_{i} y_{i}} X_{c},{ }^{O_{i} x_{i} y_{i}} Y_{c}$ - coordinates of the Gaussian function centre with respect to the $O_{i} x_{i} y_{i}$ (see fig. 1), $w_{x, i}, w_{y, i}$ - real numbers that inversely related to the width of the Gaussian function along $O_{i} x_{i}$ and $O_{i} y_{i}$ axes of the $O_{i} x_{i} y_{i}$ respectively. Put it differently the larger the $w_{x, i}$, the smaller function width along $O_{i} x_{i}$. We can derive robot coordinates ${ }^{O_{i} x_{i} y_{i}} X_{r},{ }^{O_{i} x_{i} y_{i}} Y_{r}$ using the appropriate homogeneous transform [8]: 


$$
\left(\begin{array}{c}
o_{i} x_{i} y_{i} X_{r} \\
o_{i} x_{i} y_{i} Y_{r} \\
1
\end{array}\right)=\left(\begin{array}{ccc}
\cos \gamma_{i} & \sin \gamma_{i} & -X_{i-1} \cos \gamma_{i}-Y_{i-1} \sin \gamma_{i} \\
-\sin \gamma_{i} & \cos \gamma_{i} & X_{i-1} \sin \gamma_{i}-Y_{i-1} \cos \gamma_{i} \\
0 & 0 & 1
\end{array}\right)\left(\begin{array}{c}
X_{r} \\
Y_{r} \\
1
\end{array}\right),
$$

where $\gamma_{i}$ is the angle between the line segment connecting the $(i-1)$-th and $i$-th nodal points and $O X$ axis, $X_{r}, Y_{r}$ the robot coordinates with respect to the world coordinate system $O X Y$. The robot must move from one nodal point to another and this can be done if each Gaussian function that defines attractive force has appropriate width along axis $O_{i} x_{i}$ (along line segment). More precisely one vector field must intersect with another at the appropriate Gaussian function value. Thus parameter $w_{x, i}$ is defined as:

$$
w_{x, i}=\frac{1.5 \sqrt{2}}{\sqrt{\left(X_{i}-X_{i-1}\right)^{2}+\left(Y_{i}-Y_{i-1}\right)^{2}}}=\frac{1.5 \sqrt{2}}{d_{i}},
$$

where $d_{i}$ - the distance between $(i-1)$-th and $i$-th nodal points. As it was mentioned above width of the Gaussian function along line segment, i.e. along $O_{i} x_{i}$ axis must be greater than width along $O_{i} y_{i}$ axis (transverse direction). That can be done if we choose parameter $w_{y, i}$ greater than $w_{x, i}$ :

$$
w_{y, i}=k_{w} w_{x, i},
$$

where $k_{w}>1$ - constant of proportionality, that define how Gaussian function would be narrowed along $O_{i} y_{i}$ axis.

To smooth robot motion near with the nodal points the vector fields that define orbital (or rotating) and repulsive forces are used. These vector fields act around special points - barycentres. In this research barycentres are the nodal points except the final point (goal position) for which there is only an attractive force. The orbital and repulsive force magnitude is a function of the distance between robot and barycentre. For this function, we also take default Gaussian function with the same width along all directions. The $j$-th $(j=\overline{1, m})$ orbital force magnitude $F_{o r b, j}$ then defined by the next equation:

$$
F_{o r b, j}=s_{j} F_{o r b, 0} \cdot e^{-\left[\left(w_{o, j}\left(X_{r}-X_{b, j}\right)\right)^{2}+\left(w_{o, j}\left(Y_{r}-Y_{b, j}\right)\right)^{2}\right]},
$$

where $s_{j}$ - coefficient that specifies orbital force direction: for clockwise direction $s=1$, otherwise $s=-1, F_{o r b, 0}$ orbital force basis magnitude, $w_{o, j}$ - real number that define the orbital and the repulsive force area of action with the centre at the $j$-th barycentre, $X_{b, j}, Y_{b, j}$ - the $j$-th barycentre coordinates with respect to the world coordinate system $O X Y$ (see fig. 2). A number of the barycentres is given by $m=n-1$. Figure 2 shows the vector fields of orbital forces and necessary notations.

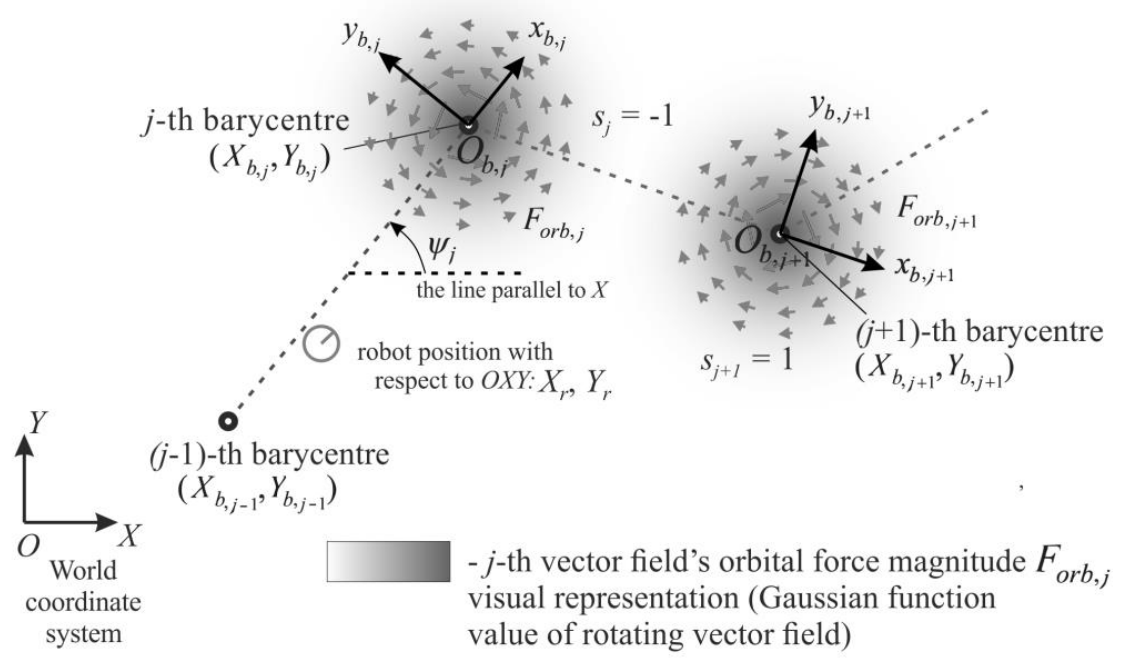

Fig. 2. Vector fields of orbital forces that acting around barycentres 
The coefficient $w_{o, j}$ can be evaluated by this equation:

$$
w_{o, j}=\frac{3}{r_{j} \sqrt{2}},
$$

where $r_{j}$ - the radius of action of the orbital force and the repulsive force. For $r_{j}$ we use the next function:

$$
r_{j}=g\left(d_{j}, d_{j+1}\right)=k_{r} \frac{d_{j} d_{j+1}}{d_{j}+d_{j+1}},
$$

where $k_{r}$ - constant of proportionality, $d_{j}$ - the distance between $(j-1)$-th and $j$-th barycentres, $d_{j+1}$ - the distance between $j$-th and $(j+1)$-th barycentres. To determine coefficient $s_{j}$ it is necessary to find $y$ coordinate of the $(j+1)$-th barycentre with respect to the $j$-th barycentre coordinate system $O_{b, j} x_{b, j} y_{b, j}$ (see fig. 2):

$$
o_{b, j} x_{b, j} y_{b, j} y_{j+1}=-\left(X_{b, j+1}-X_{b, j}\right) \sin \psi_{j}+\left(Y_{b, j+1}-Y_{b, j}\right) \cos \psi_{j},
$$

where $X_{b, j+1}, Y_{b, j+1}$ - coordinates of the $(j+1)$-th barycentre with respect to the $O X Y, \psi_{j}$ - the angle between line segment connecting $j$-th and the $(j+1)$-th barycentres and $O X$ axis. If the coordinate $O_{b, j} x_{b, j} y_{b, j} y_{j+1}<0$, then $s_{j}=-1$, else $s_{j}=1$. The $j$-th $(j=\overline{1, m})$ repulsive force magnitude $F_{r e p, j}$ defined by the next equation:

$$
F_{r e p, j}=F_{r e p, 0} \cdot e^{-\left[\left(w_{o, j}\left(X_{r}-X_{b, j}\right)\right)^{2}+\left(w_{o, j}\left(Y_{r}-Y_{b, j}\right)\right)^{2}\right]},
$$

where $F_{\text {rep }, 0}$ - the repulsive force basis magnitude. Fig. 3 shows the vector fields of repulsive forces and necessary notations.

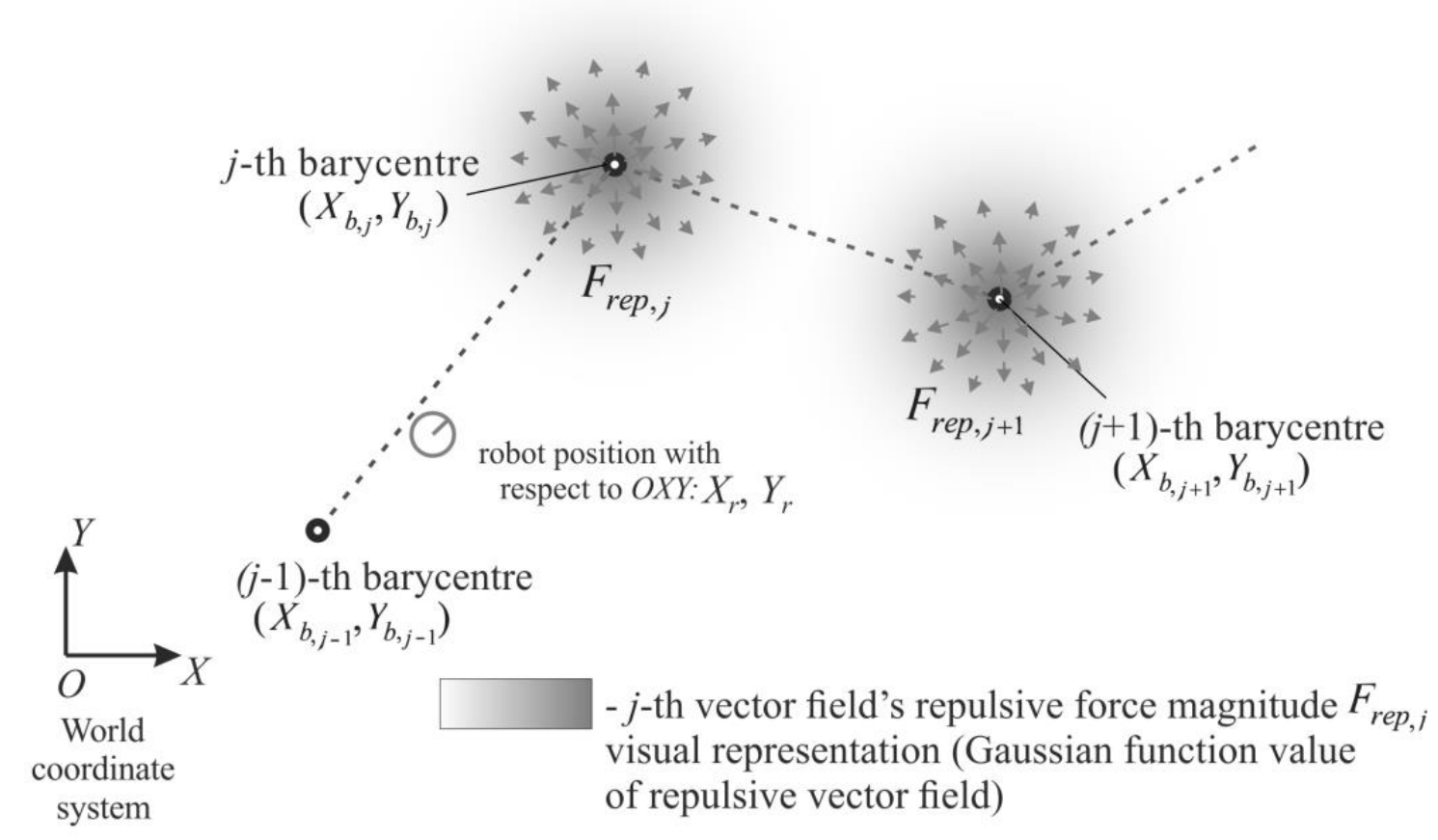

Fig. 3. Vector fields of repulsive forces that acting around barycentres

All forces act upon mobile robot but the most distant points from robot almost have no influence over a robot. Summarizing the above we can say that in each point of the two-dimensional space there are vector fields acting upon a mobile robot. The total force projection onto an $O^{\prime} x$ axis of the robot proportional to its linear velocity $V$, the total force projection onto an $O^{\prime} y$ axis of the robot proportional to its angular velocity $\omega$ (fig. 4). 


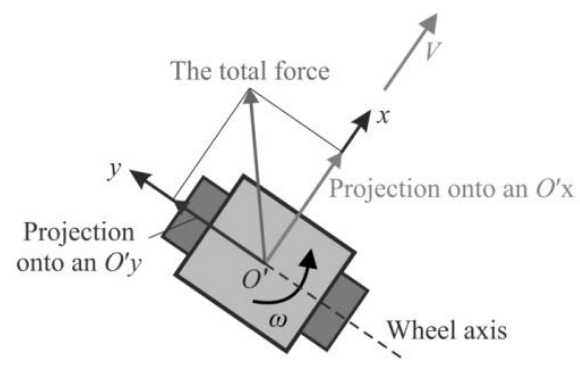

Fig. 4. The total force projections onto robot axes

To find total force projections onto axes $O^{\prime} x$ and $O^{\prime} y$ we should calculate projections of the attractive, orbital and repulsive forces onto these axes. The attractive force projections onto an axes of the robot coordinate system are defined by the next equation:

$$
\begin{aligned}
& { }^{O^{x} x y} F_{x, i}=F_{i}\left(\cos \alpha_{i} \cos \varphi+\sin \alpha_{i} \sin \varphi\right) \\
& { }^{\prime} x y F_{y, i}=F_{i}\left(\sin \alpha_{i} \cos \varphi-\cos \alpha_{i} \sin \varphi\right),
\end{aligned}
$$

where ${ }^{O^{\prime} x y} F_{x, i},{ }^{\prime} x y F_{y, i}$ - the attractive force projections onto an $O^{\prime} x$ and $O^{\prime} y$ axis respectively, $\alpha_{i}$ - the angle of $i$-th attractive force direction to the $i$-th nodal point, $\varphi$ - the angle of the robot orientation with respect to the world coordinate system $O X Y$. The repulsive force projections ${ }^{\prime} x y F_{\text {repx }, i} O^{\prime} x y F_{\text {repy,i }}$ can be found similarly except for the signs:

$$
\begin{aligned}
& { }^{O^{\prime} x y} F_{\text {repx }, i}=-F_{\text {rep }, j}\left(\cos \beta_{j} \cos \varphi+\sin \beta_{j} \sin \varphi\right) \\
& { }^{\prime}{ }^{\prime} x y F_{\text {repy }, i}=-F_{\text {rep }, j}\left(\sin \beta_{j} \cos \varphi-\cos \beta_{j} \sin \varphi\right),
\end{aligned}
$$

where $\beta_{j}$ - the angle of $j$-th repulsive force direction to the $j$-th barycentre. The orbital force projections onto axes of the robot coordinate system are defined by the equation:

$$
\begin{aligned}
& { }^{O^{\prime} x y} F_{o r b x, j}=F_{o r b, j}\left(\cos \beta_{j} \sin \varphi-\sin \beta_{j} \cos \varphi\right) \\
& { }^{\prime} x y F_{o r b y, j}=F_{o r b, j}\left(\sin \beta_{j} \sin \varphi+\cos \beta_{j} \cos \varphi\right),
\end{aligned}
$$

where ${ }^{O^{\prime} x y} F_{o r b x, j},{ }^{O^{\prime} x y} F_{o r b y, j}$ - the orbital force projections onto an $O^{\prime} x$ and $O^{\prime} y$ axis respectively.

Now we can evaluate the total force projections onto axes $O^{\prime} x$ and $O^{\prime} y$ :

$$
\begin{aligned}
& { }^{O^{\prime} x y} F_{O^{\prime} x}=\sum_{i=1}^{n}{ }^{O^{\prime} x y} F_{x, i}+\sum_{j=1}^{m}\left({ }^{O^{\prime} x y} F_{o r b x, j}+{ }^{O^{\prime} x y} F_{r e p x, j}\right) \\
& { }^{\prime} x y F_{O^{\prime} y}=\sum_{i=1}^{n}{ }^{\prime} x y F_{y, i}+\sum_{j=1}^{m}\left({ }^{O^{\prime} x y} F_{o r b y, j}+{ }^{O^{\prime} x y} F_{r e p y, j}\right) .
\end{aligned}
$$

where ${ }^{O^{\prime} x y} F_{O^{\prime} x},{ }^{O^{\prime} x y} F_{O^{\prime} y}$ - the total force projections onto axes $O^{\prime} x$ and $O^{\prime} y$. At the final step we define linear $V$ and angular $\omega$ velocities of the mobile robot:

$$
V=\frac{K_{v}}{M_{r}}{ }^{\prime} x y F_{O^{\prime} x}, \quad \omega=\frac{K_{\omega}}{J_{r}}{ }^{\prime} x y F_{O^{\prime} y},
$$

where $K_{v}$ - the linear velocity gain, $M_{r}$ - the mass of the mobile robot, $K_{\omega}$ - the angular velocity gain, $J_{r}$ - the moment of inertia of the mobile robot around vertical axis of rotation. Also for this control law we place usual limitations: if $V<V_{\min }$, then $V=V_{\min }$, if $V>V_{\max }$, then $V=V_{\max }$ and the same for the angular velocity. Here $V_{\min }$ - minimal linear velocity of the robot, $V_{\max }$ - maximum linear velocity of the robot. 
The robot motion and its trajectory are depended on not only nodal points but also on a set of main (core) parameters that were defined above: $F_{0}, k_{w}, F_{o r b, 0}, k_{r}, F_{r e p, 0}, K_{v}, K_{\omega}$.

All these parameters can have different values but one condition must always hold:

$$
F_{\text {orb }, 0} \leq F_{\text {rep }, 0}<F_{0} \text {. }
$$

If this condition is not satisfied the robot can't reach any nodal point. In the next section the most significant simulation results would be considered.

\section{Simulation results}

We tested our method for various paths with different parameters using computer simulation (MATLAB Simulink). We assume that the mobile robot has a local map of the environment and the robot control system at the higher level can generate a path from the start position to the goal position. For example, the A* algorithm can be suitable for it.

1. Consider that the mobile robot has the given set of nodal points: $(1,1.5),(2,0),(3.2,1.7),(5.2,1),(4.1,0),(3.5,0.5)$ and $(4,1)$ - coordinates of the goal position. Let $\left(X_{0}, Y_{0}\right)=(0,0)$ be the start position of the robot and $\varphi_{0}=0-$ initial orientation angle of the robot. For this example we set $F_{o r b, 0}=0$ and $F_{\text {rep, }, 0}=0$, i.e. there are only attractive forces that act on the robot. We set other parameters like so: $F_{0}=3, k_{w}=1.7, k_{r}=2, K_{v}=1.5$ and $K_{\omega}=1.2, M_{r}=10 \mathrm{~kg}$, $J_{r}=0.487{\mathrm{~kg} \times \mathrm{m}^{2}}^{2}$. Figure 5 a shows the robot trajectory and the given path, figure $5 \mathrm{~b}$ shows the actual linear and angular velocities of the robot during the motion.

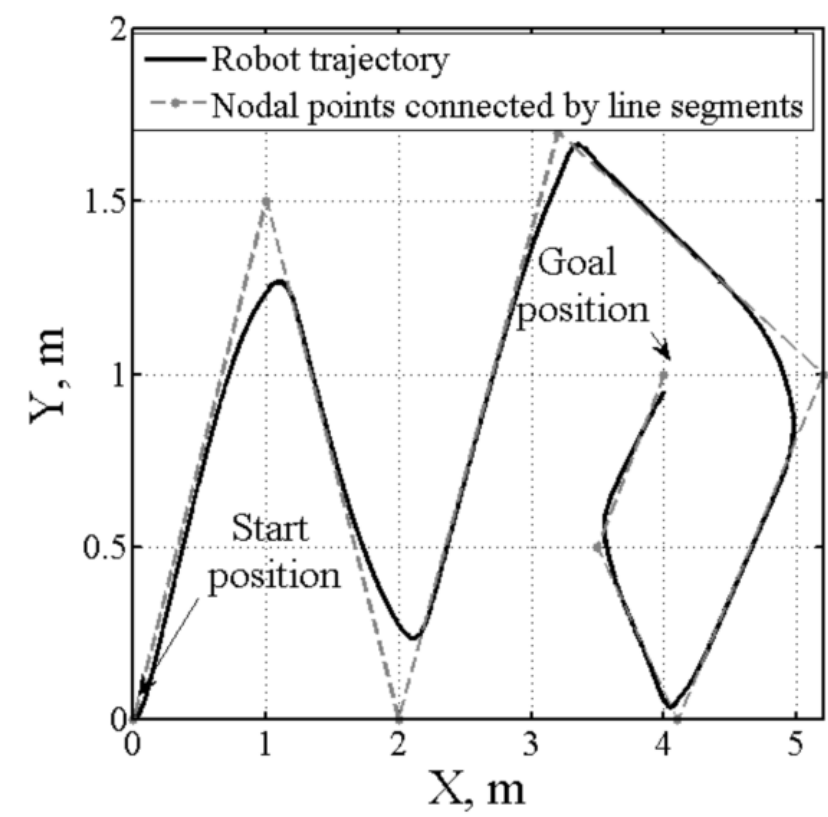

a)

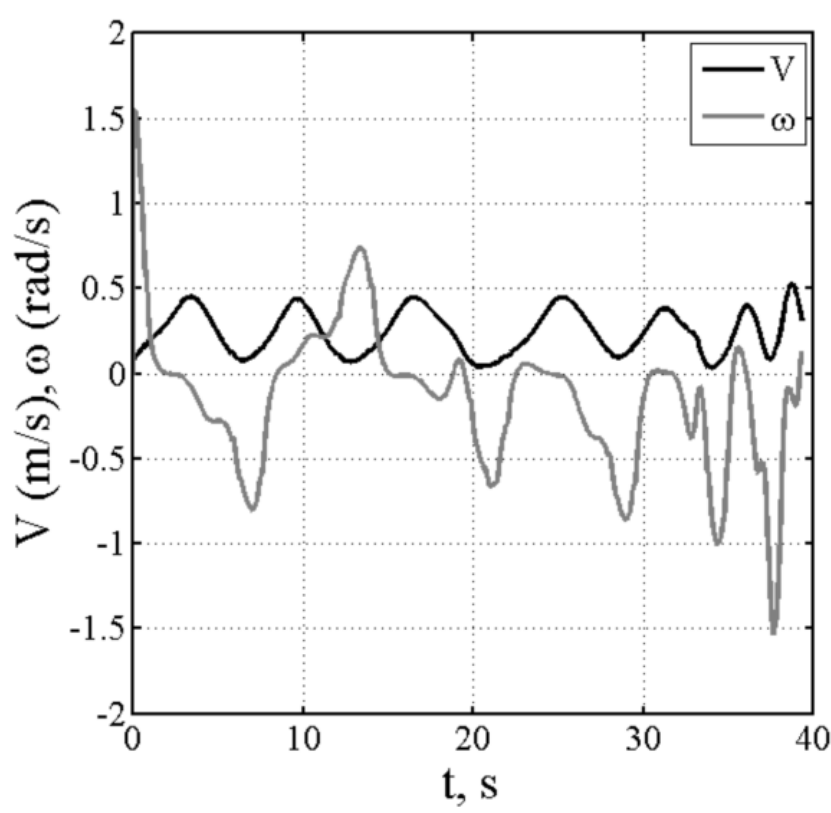

b)

Fig. 5. The robot trajectory (a) and its velocities (b) during the motion without orbital and repulsive forces

From these simulation results it is clear that the robot motion nearby the nodal point (i.e. along the path) can be successfully performed without using the orbital and repulsive forces. The robot trajectory is smooth, linear and angular velocities change continuously (see fig. 5b). Note how the linear velocity drops when the angular velocity increases. It means that robot slows down at turns and accelerates at straight-line segments of the robot path.

2. Now consider the situation when all forces are in count for the same path. The orbital and repulsive forces provide an opportunity to make robot trajectories similar to for example B-splines. In this case we set parameters with the next values: $F_{o r b, 0}=F_{r e p, 0}=1, k_{r}=2$ and other are the same. Figure 6 a shows the robot trajectory and the given path, figure $6 \mathrm{~b}$ shows the linear and angular velocities of the robot during the motion. 


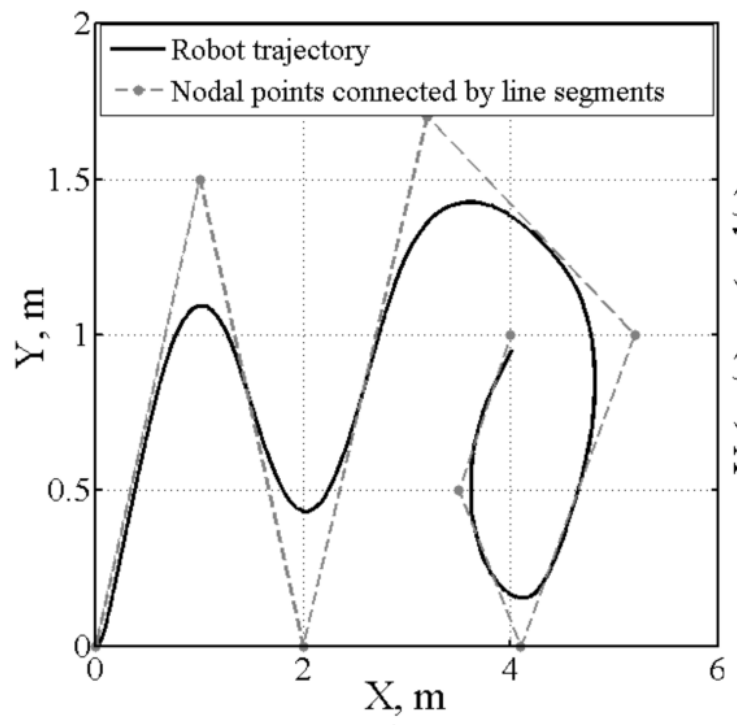

a)

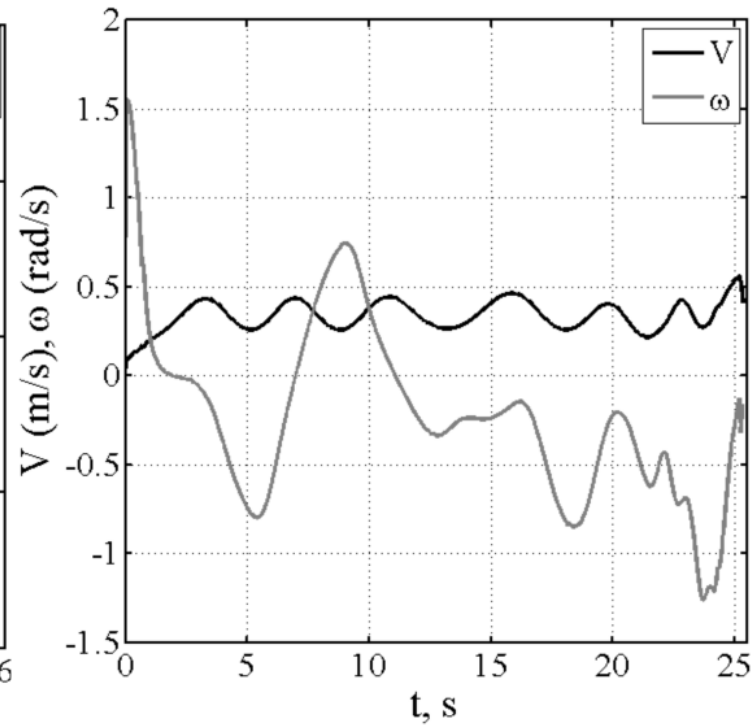

b)

Fig. 6. The robot trajectory (a) that similar to B-spline and the robot velocities (b) during the motion

One can see from this plot that the robot trajectory is similar to B-spline. Also notice that the mobile robot moves faster in comparison to previous situation (in fig. $5 \mathrm{~b}$ linear velocity decreases almost to zero, but in fig. $6 \mathrm{~b}$ linear velocity is always larger than $0.25 \mathrm{~m} / \mathrm{s}$ ).

3. Consider the following example. The mobile robot must pass through the door by consequently visiting the set of nodal points: $(1,1.5),(2.3,1.5),(2.3,0.4)$ with the $(1,0.7)$ as the start position with the orientation angle $\varphi_{0}=0$. Coordinates of the goal position are $(2.3,0.4)$. We set orbital force basis magnitude in this case as $F_{\text {orb }, 0}=0.5$ and $k_{r}=1.5$ while other parameters are same. Figure $7 \mathrm{a}$ shows the robot trajectory and the given path, figure $7 \mathrm{~b}$ shows the linear and angular velocities of the robot during the motion.

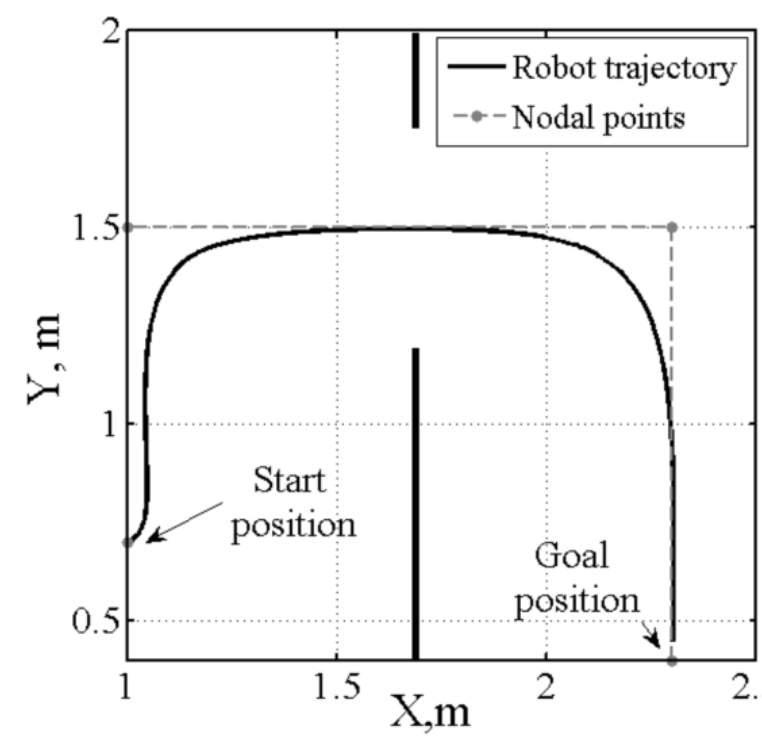

a)

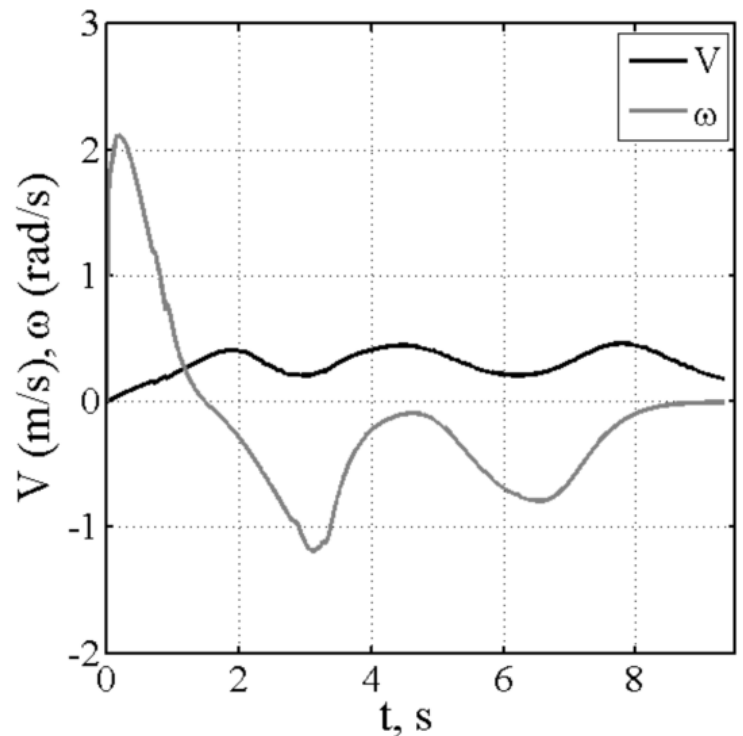

b)

Fig. 7. The robot trajectory (a) as it goes through the door and corresponding velocities (b)

Like in other situations robot velocities change smoothly and continuously. Some other results are presented in the figure 8 with the same parameters. In the fig. $8 \mathrm{a}$ the robot initial position is $\left(X_{0}, Y_{0}, \varphi_{0}\right)=(1,0,0)$, in the fig. $8 \mathrm{~b}-$ $\left(X_{0}, Y_{0}, \varphi_{0}\right)=(0,0,0)$, in the fig. $8 \mathrm{c}-\left(X_{0}, Y_{0}, \varphi_{0}\right)=(0,0, \pi / 2)$. Coordinates of the goal positions are $(1.1,5),(-1,-3)$ and $(1,5)$ respectively. 


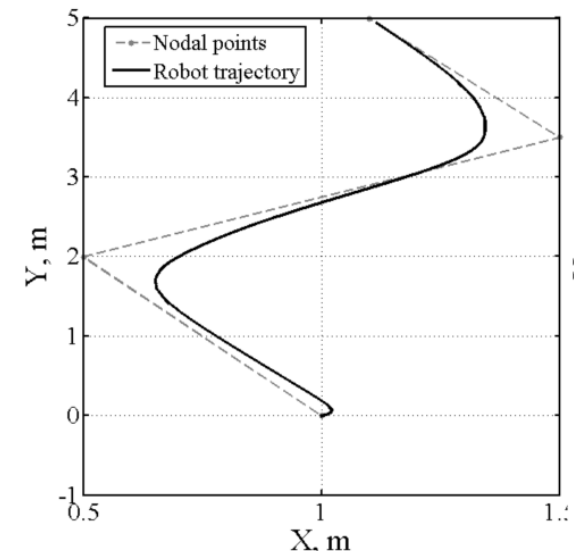

a)

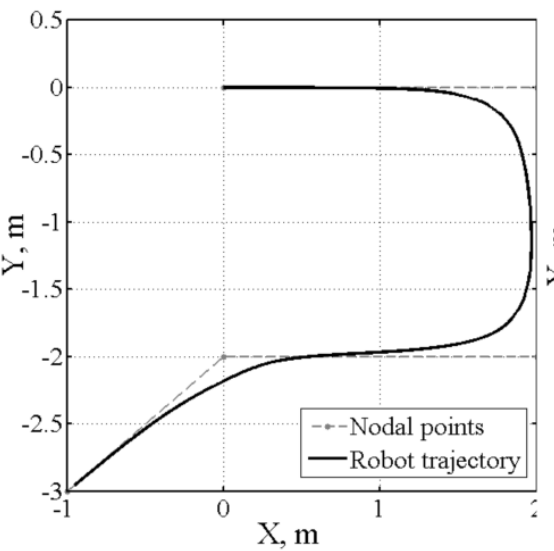

b)

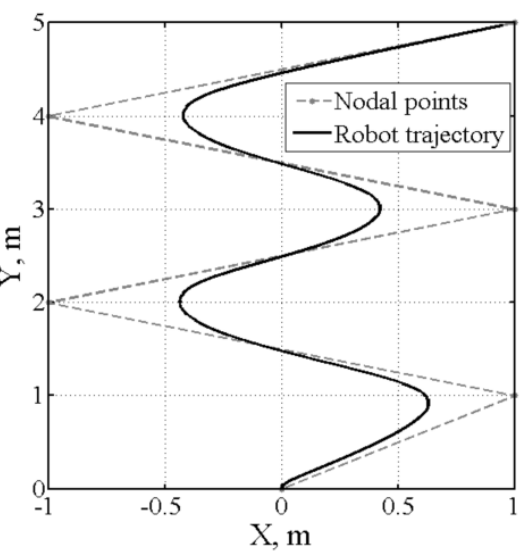

c)

Fig. 8. The robot trajectories for different paths (sets of nodal points): a), b) and c)

Simulation results analysis. The robot motion planning using vector fields gives next advantages: a differential drive mobile robot can move along complex trajectories like B-splines without their planning, robot linear and angular velocities change smoothly and continuously, the variety of robot trajectories can be achieved by using different core parameters.

\section{The application of the method to a heterogeneous modular mobile robot}

This method of motion planning is well suited to a heterogeneous modular mobile robot which concept is considered in detail in [9]. The main feature of this modular robot is based on the principle of full functionality of its modules. According to the [9] the full functionality of a mechatronic device is the ability to perform its goal function using only its own facilities for executing instructions from an external control system. This heterogeneous modular mobile robot consists of the next basic modules: a transport module that performs locomotion of the whole robot, a module-supervisor that responsible for control goals definition, the power supply module and sensory modules. Some features of the transport module control system and design were considered in [10] in which old name was used for the transport module - the motion module.

In this research we will consider the wheeled transport module and its interaction with the module-supervisor. According to the definition of the full functionality the module-supervisor gives a goal position to the transport module but verifies only result of the module performance and not the working process (except for emergency situations). Therefore the transport module control system must plan its trajectory to the goal position and perform corresponding motion. In our heterogeneous modular robot this transport module can use different strategies for motion planning. The transport module also uses data structure from a sensor module for obstacle avoidance. Note that a sensor module sends to the transport module not the usual data as array of some measurements but data structures for example, positions of lines that approximates obstacle geometry.

We use simple transport module that technically is a differential drive mobile platform. Figure 9 shows the laboratory prototype of the transport module and the modular robot.

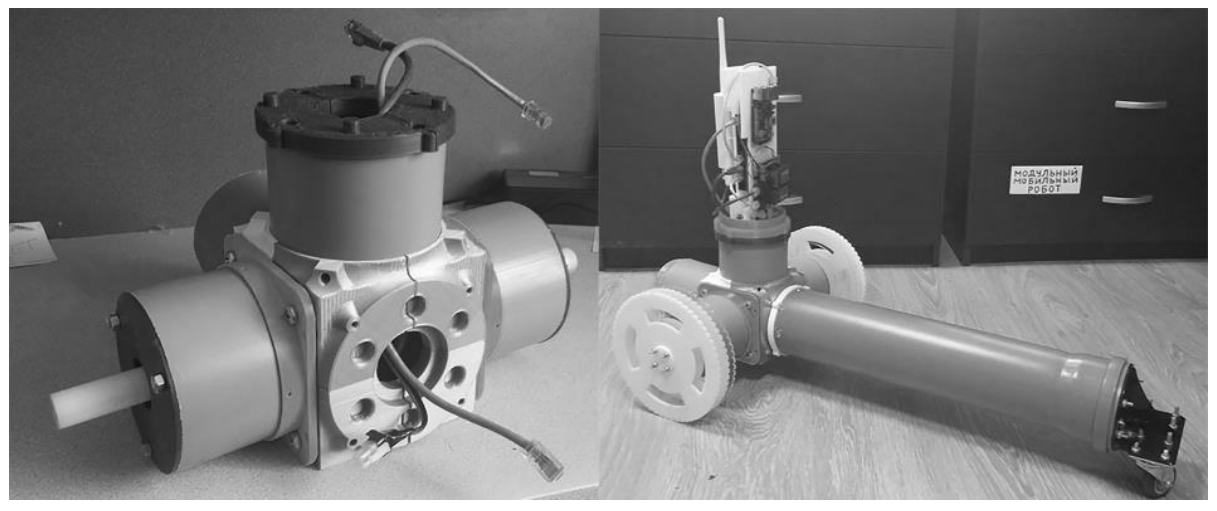

Fig. 9. The laboratory prototype of the transport module (without wheels) and modular mobile robot

Consider the interaction procedure between the transport module and the module-supervisor by the example of typical motion tasks. The modular robot must go to the goal position from the initial position in two cases: if the modulesupervisor doesn't have a map of the environment (fig. 10a) and if the module-supervisor has a map (fig. 10b). 


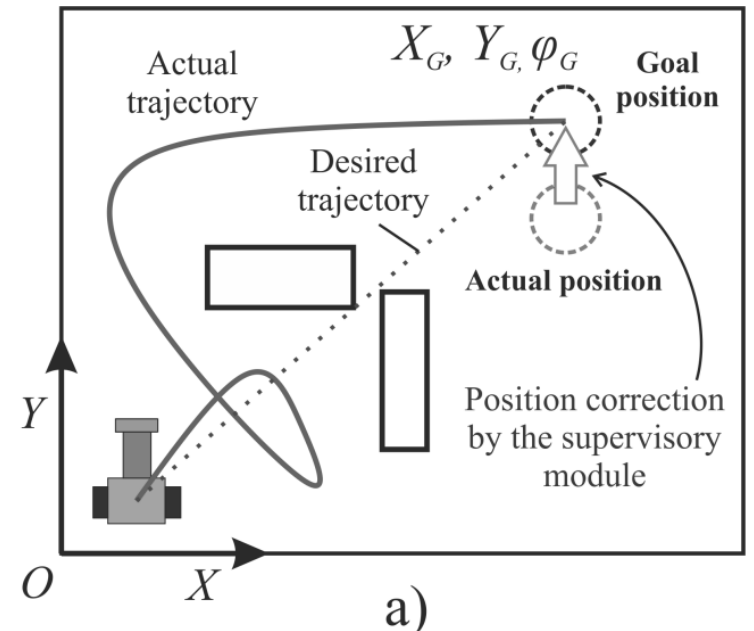

a)

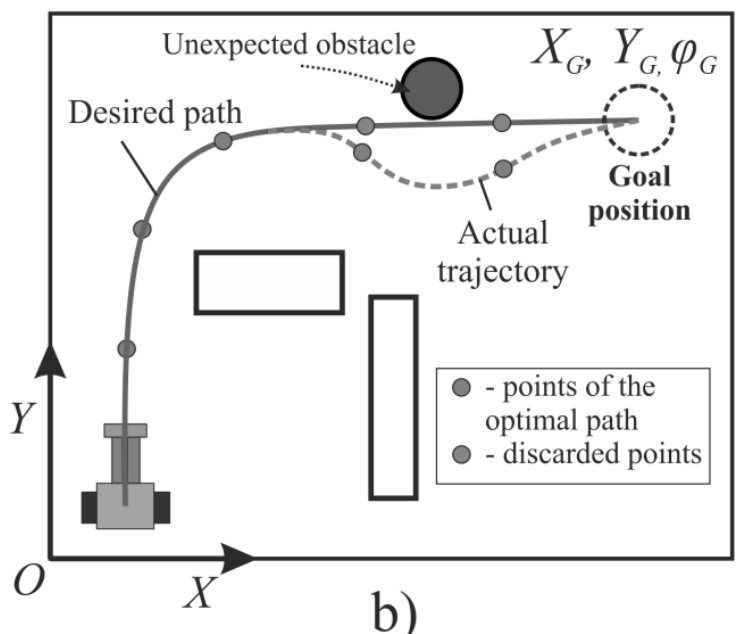

b)

Fig. 10. Examples of typical motion tasks of the mobile robot: a) - the module-supervisor doesn't have a map of the environment; b) - the module-supervisor has a map

In both cases the interaction between the transport module and the module-supervisor is defined by next sequence of actions:

1. The module-supervisor and the transport module establish the connection and begin to exchange with initial data (IP addresses of the transport module and the module-supervisor in the shared robot network, used interfaces, data conversion).

2. The module-supervisor sends to the transport module the data about goal position as set of three numbers: $X_{G}, Y_{G}-$ coordinates of the goal position in the world coordinate system, $\varphi_{G}$ - orientation angle at the goal position.

3. The transport module goes to the goal position and avoids obstacles using the data from a sensor module.

4. If the goal position is reached (or not reached) the transport module stops and sends to the module-supervisor information about the robot actual position and the message that the task was completed (or not completed).

When the robot doesn't have information about an environment (fig. 10a) the robot motion is not optimal and the arrival to the goal position is not guaranteed. In this case the module-supervisor corrects a robot position error. If the robot motion occurs in the known environment the module-supervisor can define optimal path as the set of goal positions nodal points (fig. 10b). In this case we can apply our method of motion planning using vector fields. The modulesupervisor act as robot path planner at the higher control level and the transport module control system evaluates vector fields for nodal points. If the unexpected obstacle obstructs the robot motion then the transport module is trying to avoid it by itself requesting the new goal position from the module-supervisor.

As it was mentioned above the interaction between a sensor module and the transport module is a one-way communication. While the robot moves to a goal a sensor module perform pre-processing of the measurement data and sends it to the transport module. For the localization in the environment the transport module uses the sensor data from a sensor module and an encoder data (dead reckoning).

Note that the module-supervisor doesn't control motion planning of the robot because the transport module responsible for vector fields evaluating and computation of linear and angular velocities. That distributed control is the main advantage of the full functionality of the robot modules.

\section{Conclusion}

The motion planning of the differential drive mobile robot using artificial vector fields was presented. In this method we use vector fields that produce three kinds of forces: attractive, orbital and repulsive. These forces allow a mobile robot to move along complex trajectories without their preparatory definition. In this paper we demonstrated that robot trajectories can be similar to different splines (B-splines). The smooth and continuous motion of the robot in this case is the important advantage of the method. Note that for these results there is no need to use complex control laws for path following which require constant tracking of this path.

We approved that this method is well suited to a heterogeneous modular mobile robot which modules are full functionally devices. The task of motion planning can be efficiently divided between the module-supervisor, the transport module and the sensor module. The computation load from the central processing unit of the module-supervisor is distributed between other modules according to their functions.

Future work consists of implementation of this motion planning method in the laboratory prototype of the modular mobile robot. Also we will continue our work with this method for further modifications and improvements. 


\section{Acknowledgments}

This work is supported by the Russian Foundation for Basic Research: grant 16-07-00811a, 16-07-00935a and 16-0701264a.

\section{References}

[1]. Crnokic, B.; Rezic, S. \& Pehar, S. (2016). Comparision of edge detection methods for obstacles detection in a mobile robot environment, Proceedings of the 27th DAAAM International Symposium, pp.0235-0244, B. Katalinic (Ed.), Published by DAAAM International, ISBN 978-3-902734- 08-2, ISSN 1726-9679, Vienna, Austria DOI: 10.2507/27th.daaam.proceedings.035.

[2]. Ilyukhin, Y. \& Tatarintseva, A. (2016). Control mobile robot with hybrid drive, Proceedings of the 27th DAAAM International Symposium, pp.0405-0416, B. Katalinic (Ed.), Published by DAAAM International, ISBN 978-3902734-08-2, ISSN 1726-9679, Vienna, Austria DOI: 10.2507/27th.daaam.proceedings.060.

[3]. Vinod, R.N \& Abraham, T. M. Design, simulation and implementation of cascaded path tracking controller for a differential drive mobile robot, International Conference on Advances in Computing, Communications and Informatics (ICACCI), 2015, pp. 1085-1090.

[4]. Shojaei, K.; Shahri, A.M; Tarakameh, A. and Tabibian, B. (2010). Adaptive trajectory tracking control of a differential drive wheeled mobile robot. Robotica, Vol. 29, 2010, pp. 391-402, DOI:10.1017/S0263574710000202.

[5]. Lapiere, L.; Soetanto, D. \& Pascoal A. (2006). Nonsingular path following control of unicycle in the presence of parametric modeling uncertainties. International Journal of Robust Nonlinear Control, No. 16, 2006, pp. 485-503, DOI: $10.1002 /$ rnc.1075.

[6]. Fareh, R.; Saad, M.; Khadraoui, S. and Rabie, T. (2016). Lyapunov-based tracking control for nonholonomic wheeled mobile robot. International Journal of Electrical, Computer, Energetic, Electronic and Communication Engineering, Vol. 10, No. 8, 2016, pp. 1021-1026.

[7]. Samson, C. \& Ait-Abderrahim, K. (1991). Feedback control of a nonholonomic wheeled cart in cartesian space, Proceedings of IEEE International Conference, 1991, pp. 1136-1141.

[8]. Kelly, A. (2013). Mobile Robotics, Mathematics, Models and Methods, Cambridge University Press, ISBN 978-1107-03115-9, USA.

[9]. Andreev, V; Kim, V \& Pletenev, P. (2017). The principle of full functionality - the basis for rapid reconfiguration in heterogeneous modular mobile robots, Proceedings of the 28th DAAAM International Symposium, pp.xxxxxxxx, B. Katalinic (Ed.), Published by DAAAM International, ISBN 978-3-902734-xx-x, ISSN 1726-9679, Vienna, Austria, DOI: 10.2507/28th.daaam.proceedings.xxx, in press.

[10]. Andreev, V \& Kim, V. (2016). Control system and design of the motion module of a heterogeneous modular mobile robot, Proceedings of the 27th DAAAM International Symposium, pp. 586-594, B. Katalinic (Ed.), Published by DAAAM International, ISBN 978-3-902734-08-2, ISSN 1726-9679, Vienna, Austria, DOI: 10.2507/27th.daaam.proceedings.086. 